\title{
Medidas de conforto e comunicação nas ações de cuidado de enfermagem ao paciente crítico
}

\author{
Comfort and communication measures in nursing caring actions for critically ill patients
}

Medidas de comodidad y comunicación en las acciones de atención de enfermería a pacientes críticos

\author{
Franciele Soares Pott', Taniclaer Stahlhoefer", Jorge Vinícius Cestari Felix ${ }^{\text {III, }}$ Marineli Joaquim Meier ${ }^{\text {IV }}$ \\ 'Universidade Federal do Paraná, Departamento de Enfermagem, Programa de Pós Graduação em Enfermagem \\ (Mestranda), Grupo de Pesquisa Tecnologia e Inovação em Saúde. Curitiba-PR, Brasil. \\ "Universidade Federal do Paraná, Departamento de Enfermagem, \\ Grupo de Pesquisa Tecnologia e Inovação em Saúde. Curitiba-PR, Brasil. \\ I' Universidade Federal do Paraná, Departamento de Enfermagem. Curitiba-PR, Brasil. \\ Iv Universidade Federal do Paraná, Departamento de Enfermagem, Programa de Pós-Graduação em Enfermagem, \\ Grupo de Pesquisa Tecnologia e Inovação em Saúde. Curitiba-PR, Brasil.
}

\section{Submissão: 14-04-2011 Aprovação: 06-03-2013}

\section{RESUMO}

Estudo descritivo, quantitativo, realizado em um hospital universitário de Curitiba-PR. Objetivou-se analisar as ações de cuidado realizadas em um centro de terapia semi-intensiva, na perspectiva da humanização da assistência, e avaliar a presença de medidas de conforto e comunicação na realização destas ações. A coleta de dados ocorreu mediante observação sistemática não participante. As ações de cuidado foram agrupadas, conforme sua frequência, e apresentadas em gráficos. As medidas de conforto estiveram presentes em $45 \%$ das ações de cuidado desenvolvidas e o estabelecimento de comunicação em $40 \%$ destas ações. Ainda hoje, as medidas de conforto e comunicação, traduzidas no processo de humanização do cuidado, continuam como um discurso ideal, porém muito distante da realidade dos usuários e trabalhadores da saúde.

Descritores: Cuidados de Enfermagem; Humanização da Assistência; Tecnologia.

\begin{abstract}
Descriptive, quantitative study carried out at a University Hospital in Curitiba-PR, Brazil. The objective was to analyze the caring actions performed at a semi-intensive care unit, from the perspective of the caring humanization, and also to evaluate the presence of comfort and communication measures in performing these actions. The data collection occurred under a systematic non-participant observation. The caring actions were grouped, according to its frequency, and presented in graphs. The comfort measures were present at $45 \%$ of the caring actions performed, and communication establishment was present at $40 \%$ of these actions. Even today, the comfort and communication measures, as reflected in the process of caring humanization, remain as an ideal speech. However, they are too far from reality of the health care system's users and workers.
\end{abstract}

Key words: Nursing Care; Humanization of Assistance; Technology.

\section{RESUMEN}

Estudio descriptivo con enfoque cuantitativo, realizado en un Hospital Universitario en Curitiba. Este estudio tuvo como objetivo analizar las acciones de atención realizadas en un centro de terapia semi intensiva, según la perspectiva de la humanización de la atención, y evaluar la presencia de medidas de comodidad y de la comunicación en la realización de estas acciones. La técnica de recolección de datos fue la observación sistemática sin participación. Las acciones de atención se agruparon, de acuerdo con su frecuencia, y son presentadas en gráficos. Las medidas de comodidad estuvieron presentes en el $45 \%$ de las acciones de atención desarrolladas, y la comunicación estuve presente en el $40 \%$. Incluso hoy en día, las medidas de comodidad y la comunicación, tal como se refleja en el proceso de humanización de la atención, permanecen como un discurso ideal, pero lejos de la realidad para los usuarios y profesionales de la salud.

Palabras clave: Atención de la Enfermería; Humanización de la Atención; Tecnología. 


\section{INTRODUÇÃO}

A essência da Enfermagem é o cuidar, portanto faz-se necessário que o cuidado seja eficiente e prestado de forma humanizada. O aspecto humano do cuidar, com certeza, é um dos mais difíceis de ser implementado. A rotina diária e complexa que envolve o ambiente de unidades críticas faz com que, muitas vezes, os membros da equipe de enfermagem negligenciem o tocar, conversar e ouvir o ser humano que está à sua frente.

No entanto, o cuidar não se restringe à execução de atividades técnicas, mas envolve o paciente como um todo, com histórias, sentimentos e expectativas ${ }^{(1)}$. É resgatar a importância dos aspectos emocionais, psicológicos e físicos. É ouvir o que o paciente deseja expressar, acalmar, acolher, e valorizá-lo em todas as suas dimensões(2).

Neste mesmo sentido, a humanização do cuidado, parte integrante da filosofia da Enfermagem, representa um conjunto de iniciativas capaz de conciliar a melhor tecnologia disponível com promoção de acolhimento e respeito ético-cultural ao paciente ${ }^{(2)}$, no intuito de construir uma realidade mais humana.

Ressalta-se que, ao se considerar a humanização da assistência vinculada aos avanços tecnológicos e uso de alta tecnologia, percebe-se que estas possuem características distintas; entretanto, é na interação entre elas que se almeja um resultado satisfatório ao cuidado ${ }^{(3)}$.

O avanço tecnológico na área da saúde é uma conquista, a importância da tecnologia não é algo a ser questionado, pois ela em si mesma não é benéfica nem maléfica, tudo depende da forma como a usamos(4).

Salienta-se ainda que a qualidade do cuidado depende de competência técnica, mas, também, da habilidade de interação e comunicação dos trabalhadores com os usuários ${ }^{(5)}$. Sendo assim, o cuidado é feito com e para o outro e, desta forma, a comunicação é fundamental no cuidado daqueles que vivenciam um processo de hospitalização(1), principalmente pacientes críticos e terminais.

É no desempenho de funções técnicas que a competência em comunicação deve igualar-se, em nível de importância, à competência clínica, para que o paciente receba um cuidado de alta qualidade científica e humanitária, que the propicie o direito de saber o que é feito consigo, o porquê e para que ${ }^{(6)}$. Desse modo, "a humanização passa a ser entendida como um modo de cuidado centrado na VOz do indivíduo, no respeito à sua autonomia e assim, no uso de tecnologias leves que perpassam pela escuta, apreensão e satisfação de necessidades"(7).

Diante do exposto o objetivo geral deste estudo foi analisar as ações de cuidado realizadas em um centro de terapia semi-intensiva, na perspectiva da humanização da assistência. E como objetivo específico, avaliar a presença de medidas de conforto e comunicação na realização destas ações.

\section{MATERIAL E MÉTODO}

Trata-se de um estudo descritivo, de abordagem quantitativa. A pesquisa foi desenvolvida em um Centro de Terapia
Semi-Intensiva, de um Hospital Universitário da cidade de Curitiba-PR, no período de 17 a 20 de outubro de 2008.

A técnica de coleta de dados foi a observação sistemática não participante, em que o observador entra em contato com o grupo, mas não se integra a ele, observando de forma imparcial, sem pré-julgamentos. É uma observação sistemática, consciente e dirigida a um determinado fim $^{(8)}$.

Os sujeitos do estudo foram os integrantes da equipe multiprofissional de saúde, atuantes na referida clínica durante o período de observação.

A observação sistemática não participante ocorreu em um período ininterrupto de 84 horas. Foram observados quatro plantões matutinos, quatro vespertinos e três noturnos, o que totalizou o quadro funcional da unidade. Desta etapa, participaram nove observadores previamente capacitados, divididos em plantões de 6 e 12 horas.

Para registro dos dados, foi desenvolvido um instrumento de coleta, compreendendo dados de identificação da observação, como data, leito, período, observador e iniciais do paciente, e elementos alusivos à descrição das ações de cuidados, como horário em que foi desenvolvido, duração, categorias profissionais envolvidas, além de anotações complementares referentes ao cuidado, caso fosse necessário.

As ações executadas pela equipe multiprofissional foram registradas individualmente e categorizadas quanto à presença ou ausência de comunicação e medidas de conforto, durante a realização do cuidado.

Além do instrumento de coleta, foi utilizado também um diário de campo para registro das anotações, evoluções de enfermagem e as intercorrências vivenciadas no período de observação.

Os dados foram registrados em planilha eletrônica Excel e, inicialmente, agrupados de acordo com suas características em três grupos: higiene e conforto, administração de medicamentos e cuidados complexos. Posteriormente, para proceder à análise, os mesmos foram reagrupados e classificados em duas categorias, medidas de conforto e presença de comunicação, organizados em gráficos com auxílio do programa GraphPad Prism 4 e apresentados por meio de estatística descritiva.

Os princípios éticos foram observados e seguidos com base na Resolução no 196/96, obtendo-se aprovação do Comitê de Ética do Hospital de Clínicas (CEP/HC/UFPR), sob registro CEP/HC no 1510.175/2007-08. Os participantes da pesquisa leram e assinaram o Termo de Consentimento Livre e Esclarecido, sendo-lhes assegurando o sigilo e anonimato.

\section{RESULTADOS}

Durante o período da observação sistemática não participante, foram totalizados 514 procedimentos, o que despendeu, aproximadamente, 36 horas de cuidados diretos da equipe multiprofissional.

Do total de procedimentos realizados, $90(18 \%)$ estavam relacionados à higiene e conforto, os quais demandaram 9 horas e 46 minutos de serviços de enfermagem, representando cerca de $12 \%$ das horas de observação (84 horas). 
Verificou-se que as atividades de administração de medicamentos ocorreram 82 vezes, perfazendo $16 \%$ do total de procedimentos observados. Este cuidado demandou 3 horas e 41 minutos do trabalho da equipe de enfermagem, naquele período. Os cuidados de alta complexidade como intubação orotraqueal, traqueostomia, cardioversão, atendimento a parada cardiorrespiratória (PCR), drenagem de tórax, entre outros, estiveram presentes 129 vezes no período da observação, representando $25 \%$ de todos os procedimentos, e 14 horas e 17 minutos de cuidados. Outras ações, como troca de eletrodos, troca de fixação de sonda gástrica, ajuste de oxímetro, entre outros, totalizaram 213 intervenções.

Após análise inicial, as ações mais frequentes de cuidado foram agrupadas e analisadas quanto à presença ou ausência de medidas de conforto e comunicação durante sua execução, conforme evidenciado, respectivamente, nos gráficos 1 e 2 .

Nesse processo emergiram seis grupos de maior ocorrência, os quais totalizaram 214 intervenções, a saber: higiene oral, mudança de decúbito, curativo, banho no leito, aspiração orotraqueal e administração de medicamentos.

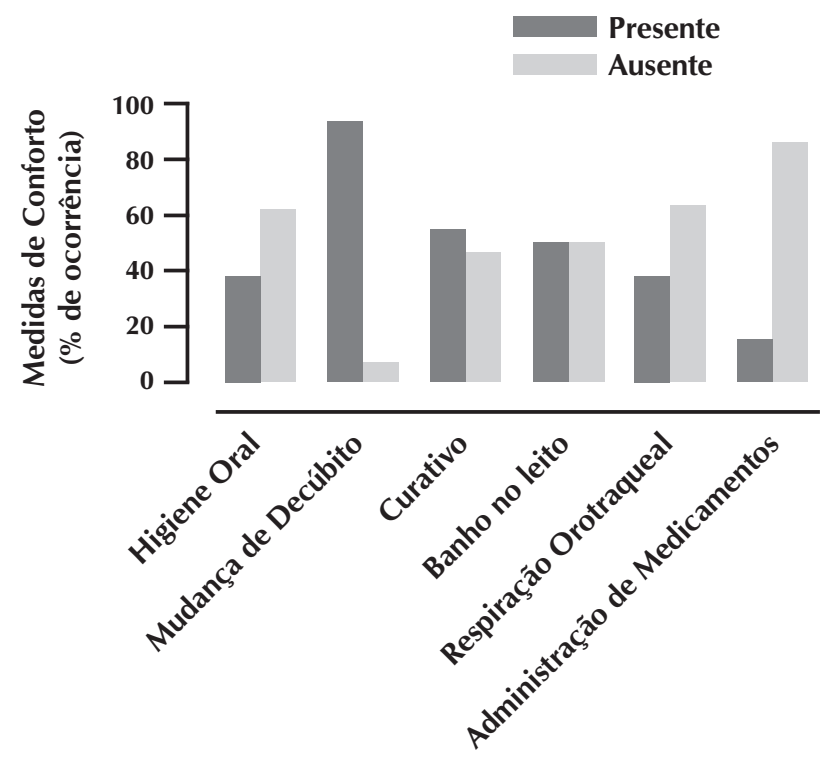

Gráfico 1 - Porcentagem de ocorrência de medidas de conforto nas ações de cuidado. Curitiba-PR, 2008.

Destaca-se que as medidas de conforto estiveram presentes em 96 (45\%) das 214 ações desenvolvidas. Ao analisar o gráfico 1, observa-se que, ao realizar mudança de decúbito, a aplicação de medidas de conforto estiveram presentes em $90 \%$ destas, considerando que esta ação, por si só, já visa o conforto do paciente.

Com relação à administração de medicamentos, verifica-se que as medidas de conforto estiveram ausentes em 66 (80\%) vezes em que este procedimento foi realizado, seguida da aspiração orotraqueal e da higiene oral, ambas com ausência das medidas de conforto em $60 \%$ (39 e 10 respectivamente) das vezes (Gráfico 1).
Ao analisar a categoria comunicação (Gráfico 2), ressalta-se que em apenas 37,5\% dos procedimentos houve o estabelecimento de alguma forma de comunicação entre profissional e paciente.

Quando se avalia a comunicação na realização do cuidado, observa-se que, na mudança de decúbito, esta esteve ausente $90 \%$ das vezes, bem como em $80 \%$ das vezes em que houve a administração de medicamentos.

De maneira geral, no tocante aos procedimentos de higiene oral, curativo, banho no leito e aspiração orotraqueal, verifica-se que a comunicação esteve ausente em 40\%; 50\%; $60 \%$ e $55 \%$ das vezes que estes foram executados, respectivamente.

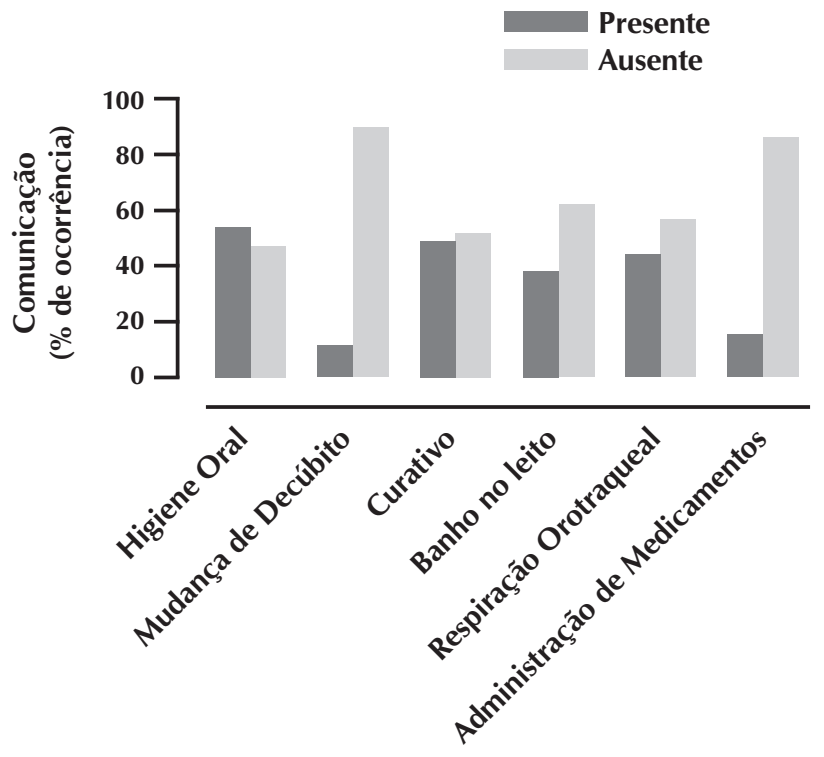

Gráfico 2 - Porcentagem de ocorrência de comunicação nas ações de cuidado. Curitiba, 2008.

\section{DISCUSSÃO}

Os procedimentos relacionados à higiene e conforto demandaram um tempo razoável da carga de trabalho da equipe. Em contraponto, estudo evidencia que a média de horas demandadas por paciente/dia em unidade de terapia semi-intensiva, nos procedimentos de higiene, é de 1 hora e 38 minutos e, para os de conforto, a média é de 2 horas $^{(9)}$. Outro trabalho que mensurou o tempo utilizado pela equipe de enfermagem para atividades assistenciais (em plantões de seis horas) também evidenciou que as menores médias de tempo despendido esteve relacionados às atividades de higiene e conforto, com um total de 3,2 minutos por paciente, o que difere sobremaneira do resultado desta pesquisa ${ }^{(10)}$.

Por outro lado, vale ressaltar que, conforme o quadro clínico em que o paciente se apresenta, procedimentos ora considerado simples, como a movimentação de articulações para a higienização do corpo tornam-se demorados em função das condições do paciente ${ }^{(11)}$, o que justifica maior consumo de tempo para esse procedimento. 
Outra atividade que merece destaque é a administração de medicamentos, elemento decisivo no processo de restabelecimento do indivíduo. Em função da gravidade do quadro clínico e do nível de dependência do paciente internado neste setor, o número de fármacos administrados é significativo, sendo este, muitas vezes um procedimento complexo, como a administração por via parenteral. Isto requer dos profissionais que os administram competências específicas, bem como extrema atenção para que esta prática seja livre de erros.

A administração de medicamentos é uma das funções assistenciais mais realizadas pela equipe de enfermagem (auxiliares e técnicos de enfermagem), sendo o enfermeiro, o líder da equipe e o responsável pelo processo de administração de medicamentos e de cuidados ao paciente que se encontra em terapia medicamentosa ${ }^{(12)}$. A Classificação das Intervenções de Enfermagem cita algumas intervenções essenciais ao cuidado a pacientes críticos, dentre as quais, a administração de medicamentos intravenosos ${ }^{(13)}$. Sendo assim, o estabelecimento de rotinas, padronizações, bem como conhecimento e a atenção acerca do tema são fatores indispensáveis à qualidade deste procedimento.

Os cuidados de alta complexidade estiveram presentes 129 vezes no período da observação. Procedimentos de alta complexidade destinados a pacientes críticos demandam alta carga de trabalho da equipe de enfermagem e 100\% deles requer administração de medicamentos ${ }^{(14)}$. Além do mais, possuem necessidades específicas de cuidados, que devem ser atendidas de modo que não haja riscos e falhas desnecessárias, bem como, possam, não só sobreviver, mas viver com qualidade de $v i^{(15)}$. A complexidade dos pacientes desta clínica é evidente devido ao tipo de ações realizadas, todas requerem domínio teórico / prático e, tecnológico para seu desenvolvimento.

\section{Medidas de conforto}

O termo conforto é, empregado em diferentes contextos da prática de enfermagem, e faz parte da linguagem usual da equipe, sendo considerado como um componente do cuidar $^{(16)}$.

Neste estudo, as medidas de conforto estiveram presentes em 45\% (96) das ações desenvolvidas. No grupo mudança de decúbito as medidas de conforto estiveram presentes em $90 \%$ das vezes em que este procedimento foi realizado. Em contraponto, em $80 \%$ das vezes em que ocorreu administração de medicamentos, nenhuma medida de conforto foi aplicada ao paciente.

Administrar medicamentos prescritos é um papel fundamental à maioria das equipes de enfermagem. Entretanto, não deve ser visto como uma tarefa mecânica a ser executada, requer pensamento e o exercício de juízo profissional. Ao privilegiar exclusivamente a técnica em detrimento ao ser humano, se fortalece uma prática desumana, promovendo uma assistência ao indivíduo e à sociedade inferior de que são merecedores ${ }^{(15)}$.

As ações higiene oral e aspiração orotraqueal foram desenvolvidas sem aplicação de medidas de conforto em $60 \%$ das situações. Cuidados com o tempo de aspiração, execução de técnica de modo a não traumatizar as vias aéreas, proteção do paciente contra secreções, entre outras, não foram consideradas durante a realização das técnicas. Sabe-se que as ações supracitadas visam, sobretudo, o conforto ao paciente.

Desta forma, algumas medidas podem/devem ser empregadas com vista a minimizar possíveis desconfortos causados por estes procedimentos. Ao se considerar a aspiração de vias aéreas, deve-se evitar aspirações desnecessárias, longas e traumáticas, além de observar o paciente quanto a complicações advindas de um longo período de aspiração tais como: apnéia, cianose, respiração curta e hipotensão ${ }^{(17)}$.

A prática de promover medidas de conforto é inerente à profissão do enfermeiro, e assim, imprescindível ao cuidado humanizado e de qualidade ao paciente, no entanto muitas vezes é minimizada frente às tecnologias presentes em ambientes complexos. Ressalta-se sua relevância no restabelecimento da saúde do indivíduo, uma vez que é por meio de medidas de conforto que os enfermeiros e sua equipe promovem reforço, esperança, consolo, apoio, encorajamento e assistência de qualidade. Ainda, proporciona melhor interação enfermeiro-paciente, bem como, possibilita o estabelecimento de vínculo efetivo, traduzido em confiança por parte do indivíduo cuidado.

Além de oferecer bem-estar físico, as medidas de conforto abarcam as demais dimensões inerentes ao indivíduo, como a dimensão psicoespiritual (que pertence à conscientização interna do eu, incluindo estima, conceito, sexualidade e significado na vida do indivíduo; pode também abranger um relacionamento do indivíduo com uma ordem mais alta ou ser superior); a ambiental (que pertence ao cenário externo da experiência humana, abrangendo luz, barulho, ambiente, cor, temperatura e elementos naturais versus sintéticos); e a dimensão social (que pertence às relações interpessoais, familiares e sociais) ${ }^{(18)}$.

O conforto torna-se fundamental quando relacionado à prática da enfermagem como um objetivo a ser atingido pela assistência, sendo visto, portanto, como um resultado desejado para o cuidado do paciente, com vistas a suprir expectativas e necessidades de cada indivíduo cuidado, e assim, alcançar uma assistência mais humana e singular.

\section{Comunicação}

Percebe-se que o estabelecimento de comunicação esteve presente em $37,5 \%$ dos procedimentos executados pela equipe multiprofissional. Entretanto, é evidente a não comunicação durante as ações de mudança de decúbito e administração de medicamentos.

Ao considerar que estas ações foram destinadas a pacientes críticos/sedados, muitos profissionais acreditam que a comunicação entre eles e paciente não é necessária, pois devido à sedação não compreendem, nem mesmo ouvem o que acontece ao seu redor. No entanto, estudos demonstram que o comprometimento de algumas funções cerebrais e sensoriais não implica necessariamente na inexistência perceptual; a diferença está na possibilidade de expressão do que é percebido $^{(19)}$. Ainda, a audição parece ser o último sentido que é perdido, e tal afirmação é sustentada por meio dos relatos de pessoas que retornaram desse estado ${ }^{(20)}$. 
É importante salientar que, além da comunicação verbal, expressa pela fala e escrita, há a comunicação não verbal, a qual abrange gestos, silêncio, expressões faciais, postura corporal, entre outros ${ }^{(21)}$. Desta forma, o enfermeiro utiliza da comunicação não verbal, expressa pelo toque, carinho, como um instrumento que permite sua interação com o paciente sedado, de modo que esta expressão proporcione ao paciente segurança e conforto, ao sentir-se não apenas como um objeto de trabalho, mas sim um ser humano possuidor de sentimentos.

A ausência de comunicação, em suas distintas formas, durante as ações de cuidado nos remete a um dilema ético/moral no qual se negligencia a dimensão psicoespiritual e social do indivíduo cuidado, que embora impossibilitado de se expressar, ainda possui suas peculiaridades, seus medos e anseios.

Outro fator a ser pontuado, é a mecanização dos cuidados diários, o que evidencia que muitos profissionais ainda se detêm apenas ao tratamento e cura do corpo doente, sem considerar as demais dimensões que envolvem o indivíduo cuidado. Tal achado vai ao encontro da literatura, em trabalho realizado ao demonstrar que o cuidado efetuado em ambiente crítico, por exigir alta tecnicidade, pode tornar-se mecânico devido à alta complexidade de equipamentos e tecnologia(21).

Estes fatores, portanto, favorecem um comportamento da equipe pouco comprometido com os sentimentos dos doentes e seus familiares, resultando na desvalorização da assistência humanizada.

No entanto, ao compreender tecnologia como a interação do saber e do fazer, mediante um processo reflexivo, que necessita da presença humana e se concretiza no ato de cuidar ${ }^{(22)}$, permite-se a interação entre profissional, tecnologia e indivíduo cuidado, com vista a uma assistência humana e de qualidade.

A literatura aponta a comunicação como instrumento mediador da humanização da assistência, utilizada no processo de trabalho do enfermeiro, de forma a estabelecer um relacionamento efetivo, que possibilite a transmissão de um sentimento de confiança, tranqüilidade, por meio do diálogo, da escuta sensível e da conversa, capazes de transformar a posição de insegurança e medo do cliente ${ }^{(23)}$. Neste sentido, o estabelecimento de uma relação para além do cuidado físico, por meio da humanização das ações desenvolvidas, favorece um processo de recuperação com qualidade ${ }^{(24)}$.

Além do mais, o processo de comunicar-se efetivamente demonstra respeito do ser cuidador ao ser cuidado e se traduz em estabelecer um relacionamento efetivo com o indivíduo. Sua aplicação diária proporciona maneiras de inovar na busca de transpor as dificuldades enfrentadas à efetivação da comunicação.

\section{CONSIDERAÇÕES FINAIS}

Embora sejam temas atuais e constantemente discutidos na literatura científica, ainda hoje, as medidas de conforto e comunicação, traduzidas no processo de humanização do cuidado, continuam como um discurso ideal, porém muito distante da realidade dos usuários e trabalhadores da saúde.

Conforme demonstra esta pesquisa, o aspecto humano do cuidado é desconsiderado durante a assistência dispensada ao indivíduo, o que pode estar relacionado às altas tecnologias que permeiam os ambientes críticos. O distanciamento entre equipe, paciente e familiar compromete a qualidade da assistência, remetendo ao paciente o papel de objeto a ser manipulado.

Assim, sugere-se uma articulação entre o cuidar e a tecnologia, de modo que a competência tecnológica seja uma expressão de cuidado, mediante valorização da interação entre o profissional, o paciente e a tecnologia - uma experiência efetiva de cuidado ${ }^{(25)}$.

Desta forma, destaca-se que estratégias devem ser pensadas, com o intuito de proporcionar uma prática de enfermagem mais humana, a qual visualize o indivíduo, não apenas como mais um, e sim como um ser possuidor de experiências próprias, mesmo quando estas experiências não possam ser expressas por palavras.

Para tanto, faz-se necessário um processo reflexivo, acerca de valores e princípios morais e éticos, que norteiem e conduzam o cuidado de Enfermagem e/ou multiprofissional, com vistas a produzir uma realidade mais humana.

\section{REFERÊNCIAS}

1. Campos ACV, Dutra BS, Borges CM, Souza AB, Santos VH. O cuidado humanizado sob a percepção dos enfermeiros. Enferm Rev 2012;15(1).

2. Mendes JR, Spíndola TS, Mota GMC. Percepção de pacientes sobre a equipe de enfermagem acerca da humanização em terapia intensiva. Rev Enferm UFPI 2012;1(3):182-7.

3. Bedin E, Ribeiro LBM, Barreto RASS. Humanização da assistência de enfermagem em centro cirúrgico. Rev Eletrônica Enferm [periódico na internet]. 2004 [acesso em 08 Abr 2010]. Disponível em: www.fen.ufg.br

4. Cunha PJ, Zagonel IPS. As relações interpessoais nas ações de cuidar em ambiente tecnológico hospitalar. Acta Paul Enferm 2008;21(3).
5. Nonino, EAPM, Anselmi ML, Dalmas JC. Avaliação da qualidade do procedimento curativo em pacientes internados em um Hospital Universitário. Rev Latino-Am Enferm 2008 [acesso em 26 fev 2010]. Disponível em www.eerp.usp.br/rlae

6. Stefanelli MC, Carvalho EC. A comunicação nos diferentes contextos da enfermagem. 2.ed. Barueri, SP: Editora Manole; 2012.

7. Fontana RT. Humanização no processo de trabalho em enfermagem: uma reflexão. Rev Rene 2010;11(1):200-207.

8. Marconi MA, Lakatos EM. Fundamentos de metodologia científica. 6.ed. São Paulo: Atlas; 2005.

9. Wolff LDG, Mazur CS, Wiezbick C, et al. Dimensionamento de pessoal de enfermagem na unidade semi-intensiva 
de um hospital universitário de Curitiba. Cogitare Enferm 2007;12(2): 171-82.

10. Moreno FN, Haddad MCL, Vannuchi MTO, Jenal S, Girotti SKO. Mensuração de horas de trabalho do enfermeiro em hospital filantrópico terciário. Cogitare Enferm 2012;17(1):50-6

11. Oliveira RA, coordenador. Cuidado Paliativo. São Paulo: Conselho Regional de Medicina de São Paulo; 2008.

12. Azevedo Filho FM, Soares IMM, Soares CSRS, Fazendeiro PG, Paranaguá TTB, Bezerra, ALQ. Administração de medicamentos: conhecimento de enfermeiros do setor de urgência e emergência. Enferm Glob 2012;11(26):70-85.

13. Bulechek GM, Butcher HK, Dochterman JM. Classificação das Intervenções de Enfermagem- NIC. 5. ed. Rio de Janeiro: Elsevier; 2010.

14. Inoue KC, Matsuda LM. Dimensionamento de pessoal de enfermagem em unidade de terapia intensiva para adultos. Acta Pau Enferm 2010;23(3):379-84.

15. Lopes BC, Vargas MAO, Azeredo NSG, Behenck A. Erros de medicação realizados pelos técnicos de enfermagem na UTI: Contextualização da problemática. Enferm Foco 2012;3(1):16-21.

16. Louro TQ, Silva RCL, Moura LF, Machado DA. A terapia intensiva e as tecnologias como marca registrada. Rev Pesq Cuid Fundam 2012;4(3):2465-82.

17. Padilha, KG. Ocorrências iatrogênicas em unidade de terapia intensiva (UTI): análise dos fatores relacionados. Rev Paul Enferm 2006;25(1):18-23.
18. Araujo AC, Spier MES. Sentimento da equipe de enfermagem durante a aspiração de uma traqueostomia. In: Anais 17.Congresso de iniciação científica; 2008 nov 11-14; Pelotas, Brasil. Rio Grande do sul: UFPEL; 2008. p.1-5.

19. Mussi FC, Friedlander MR, Neves-Arruda E. Os significados da palavra conforto segundo a perspectiva do paciente com infarto agudo do miocárdio. Rev Latino-Am Enferm 1996;4(3):19-39.

20. Zinn GR, Silva MJP, Telles SCR. Comunicar-se com o paciente sedado: vivência de quem cuida. Rev Latino-Am Enferm 2003;11(3):326-332.

21. Puggina ACG, Silva MJP. Sinais vitais e expressão facial de pacientes em estado de coma. Rev Bras Enferm 2009;62(3):435-441.

22. Siqueira AB, Filipini R, Posso MBS, Fiorano AMM, Gonçalves SA. Relacionamento enfermeiro, paciente e família: fatores comportamentais associados a qualidade da assistência. Arq Med ABC 2006;31(2):73-7.

23. Meier MJ. Tecnologia em enfermagem: desenvolvimento de um conceito. Florianópolis. Tese [Doutorado em Enfermagem] - Universidade Federal de Santa Catarina; 2004.

24. Caron OAF, Silva IA. Parturiente e equipe obstétrica: a difícil arte da comunicação. Rev. Latino-Am Enferm 2002;10(4):485-492.

25. Halldórsdóttir S. Implications of the caring competence dichotomy. In: Thorne SE, Hayes VE, editors. Nursing praxis: Knowledge and action; 1997. p.105-124. 